\title{
Inclusion properties of certain subclasses of analytic functions defined by generalized Sălăgean operator
}

ABstract. Let $A$ denote the class of analytic functions with the normalization $f(0)=f^{\prime}(0)-1=0$ in the open unit $\operatorname{disc} U=\{z:|z|<1\}$. Set

$$
f_{\lambda}^{n}(z)=z+\sum_{k=2}^{\infty}[1+\lambda(k-1)]^{n} z^{k} \quad\left(n \in N_{0} ; \lambda \geq 0 ; z \in U\right),
$$

and define $f_{\lambda, \mu}^{n}$ in terms of the Hadamard product

$$
f_{\lambda}^{n}(z) * f_{\lambda, \mu}^{n}=\frac{z}{(1-z)^{\mu}} \quad(\mu>0 ; z \in U) .
$$

In this paper, we introduce several subclasses of analytic functions defined by means of the operator $I_{\lambda, \mu}^{n}: A \longrightarrow A$, given by

$$
I_{\lambda, \mu}^{n} f(z)=f_{\lambda, \mu}^{n}(z) * f(z) \quad\left(f \in A ; n \in N_{0} ; \lambda \geq 0 ; \mu>0\right) .
$$

Inclusion properties of these classes and the classes involving the generalized Libera integral operator are also considered.

1. Introduction. Let $A$ denote the class of functions of the form:

$$
f(z)=z+\sum_{k=2}^{\infty} a_{k} z^{k}
$$

2000 Mathematics Subject Classification. 30C45.

Key words and phrases. Analytic, Hadamard product, starlike, convex. 
which are analytic in the open unit disc $U=\{z:|z|<1\}$. If $f$ and $g$ are analytic in $U$, we say that $f$ is subordinate to $g$, written $f \prec g$ or $f(z) \prec g(z)$, if there exists a Schwarz function $w(z)$, which (by definition) is analytic in $U$ with $w(0)=0$ and $|w(z)|<1$ for all $z \in U$, such that $f(z)=g(w(z)), z \in U$. For $0 \leq \eta<1$, we denote by $S^{*}(\eta), K(\eta)$ and $C$ the subclasses of $A$ consisting of all analytic functions which are, respectively, starlike of order $\eta$, convex of order $\eta$ and close-to-convex of order $\eta$ in $U$ (see, e.g., Srivastava and Owa [11]).

For $n \in N_{0}=N \cup\{0\}$, where $N=\{1,2, \ldots\}, \lambda \geq 0$ and $f$ given by (1.1), we consider the generalized Sălăgean operator defined as follows:

$$
D_{\lambda}^{n} f(z)=z+\sum_{k=2}^{\infty}[1+\lambda(k-1)]^{n} a_{k} z^{k} \quad(z \in U) .
$$

The operator $D_{\lambda}^{n}$ was introduced and studied by Al-Oboudi [1] which reduces to the Sălăgean differential operator [10] for $\lambda=1$.

Let $S$ be the class of all functions $\phi$ which are analytic and univalent in $U$ and for which $\phi(U)$ is convex with $\phi(0)=1$ and $\operatorname{Re}\{\phi(z)\}>0(z \in U)$. The Hadamard product (or convolution) $f * g$ of two analytic functions $f(z)=\sum_{k=0}^{\infty} a_{k} z^{k}$ and $g(z)=\sum_{k=0}^{\infty} b_{k} z^{k}$ is given by

$$
(f * g)(z)=\sum_{k=0}^{\infty} a_{k} b_{k} z^{k} .
$$

Making use of the principle of subordination between analytic functions, we introduce the subclasses $S^{*}(\eta ; \phi), K(\eta ; \phi)$ and $C(\eta, \delta ; \phi, \psi)$ of the class $A$ for $0 \leq \eta, \delta<1$ and $\phi, \psi \in S$ (cf., [3], [5] and [7] ), which are defined by

$$
\begin{gathered}
S^{*}(\eta ; \phi)=\left\{f \in A: \frac{1}{1-\eta}\left(\frac{z f^{\prime}(z)}{f(z)}-\eta\right) \prec \phi(z)(z \in U)\right\}, \\
K(\eta ; \Phi)=\left\{f \in A: \frac{1}{1-\eta}\left(1+\frac{z f^{\prime \prime}(z)}{f^{\prime}(z)}-\eta\right) \prec \phi(z)(z \in U)\right\}
\end{gathered}
$$

and

$$
\begin{gathered}
C(\eta, \delta ; \phi, \psi)=\left\{f \in A: \exists g \in S^{*}(\eta ; \phi) \text { s. t. } \frac{1}{1-\delta}\left(\frac{z f^{\prime}(z)}{g(z)}-\delta\right) \prec \psi(z)\right. \\
(z \in U)\} .
\end{gathered}
$$

We note that, for special choices for the functions $\phi$ and $\psi$ involved in these definitions, we can obtain the well-known subclasses of $A$. For example, we have

$$
S^{*}\left(\eta ; \frac{1+z}{1-z}\right)=S^{*}(\eta), K\left(\eta ; \frac{1+z}{1-z}\right)=K(\eta)
$$


and

$$
C\left(0,0 ; \frac{1+z}{1-z}, \frac{1+z}{1-z}\right)=C
$$

Setting

$$
f_{\lambda}^{n}(z)=z+\sum_{k=2}^{\infty}[1+\lambda(k-1)]^{n} z^{k} \quad\left(n \in N_{0} ; \lambda \geq 0\right),
$$

we define the function $f_{\lambda, \mu}^{n}$ in terms of the Hadamard product by

$$
f_{\lambda}^{n}(z) * f_{\lambda, \mu}^{n}(z)=\frac{z}{(1-z)^{\mu}} \quad(\mu>0 ; z \in U) .
$$

We now introduce the operator $I_{\lambda, \mu}^{n}: A \longrightarrow A$, which is defined here by

$$
\begin{gathered}
I_{\lambda, \mu}^{n} f(z)=f_{\lambda, \mu}^{n}(z) * f(z)=z+\sum_{k=2}^{\infty} \frac{(\mu)_{k-1}}{(k-1) ![1+\lambda(k-1)]^{n}} a_{k} z^{k} \\
\left(f \in A ; n \in N_{0} ; \lambda \geq 0 ; \mu>0\right),
\end{gathered}
$$

where $(\theta)_{k}$ is the Pochhammer symbol defined, in terms of the Gamma function $\lceil$, by

$$
(\theta)_{k}=\frac{\lceil(\theta+k)}{\lceil(\theta)}=\left\{\begin{array}{lr}
1 & (k=0, \theta \in C \backslash\{0\}), \\
\theta(\theta+1) \ldots(\theta+k-1) & (k \in N, \theta \in C) .
\end{array}\right.
$$

We note that $I_{1,2}^{1} f(z)=f(z)$ and $I_{0,2}^{0} f(z)=z f^{\prime}(z)$.

From (1.4), we obtain the following relations:

$$
\lambda z\left(I_{\lambda, \mu}^{n+1} f(z)\right)^{\prime}=I_{\lambda, \mu}^{n} f(z)-(1-\lambda) I_{\lambda, \mu}^{n+1} f(z) \quad(\lambda>0)
$$

and

$$
z\left(I_{\lambda, \mu}^{n} f(z)\right)^{\prime}=\mu I_{\lambda, \mu+1}^{n} f(z)-(\mu-1) I_{\lambda, \mu}^{n} f(z) .
$$

Next, by using the operator $I_{\lambda, \mu}^{n}$, we introduce the following classes of analytic functions for $\phi, \psi$ :

and

$$
\begin{gathered}
S_{\lambda, \mu}^{n}(\eta ; \phi)=\left\{f \in A: I_{\lambda, \mu}^{n} f(z) \in S^{*}(\eta ; \phi)\right\}, \\
K_{\lambda, \mu}^{n}(\eta ; \phi)=\left\{f \in A: I_{\lambda, \mu}^{n} f(z) \in K(\eta ; \phi)\right\}
\end{gathered}
$$

$$
C_{\lambda, \mu}^{n}(\eta, \delta ; \phi, \psi)=\left\{f \in A: I_{\lambda, \mu}^{n} f(z) \in C(\eta, \delta ; \phi, \psi)\right\} .
$$

We also note that

$$
f(z) \in K_{\lambda, \mu}^{n}(\eta ; \phi) \Longleftrightarrow z f^{\prime}(z) \in S_{\lambda, \mu}^{n}(\eta ; \phi) .
$$

In particular, we set

$$
S_{\lambda, \mu}^{n}\left(\eta ;\left(\frac{1+A z}{1+B z}\right)^{\alpha}\right)=S_{\lambda, \mu}^{n}(\eta ; A, B ; \alpha) \quad(0<\alpha \leq 1 ;-1 \leq B<A \leq 1)
$$


and

$K_{\lambda, \mu}^{n}\left(\eta ;\left(\frac{1+A z}{1+B z}\right)^{\alpha}\right)=K_{\lambda, \mu}^{n}(\eta ; A, B ; \alpha) \quad(0<\alpha \leq 1 ;-1 \leq B<A \leq 1)$.

We note that for $\lambda=1$ in the above classes, we obtain the following classes $S_{\mu}^{n}(\eta ; \phi), K_{\mu}^{n}(\eta ; \phi)$ and $C_{\mu}^{n}(\eta, \delta ; \phi, \psi)$.

In this paper, we investigate several inclusion properties of the classes $S_{\lambda, \mu}^{n}(\eta ; \phi), K_{\lambda, \mu}^{n}(\eta ; \phi)$ and $C_{\lambda, \mu}^{n}(\eta, \delta ; \phi, \psi)$ associated with the operator $I_{\lambda, \mu}^{n}$. Some applications involving these and other classes of integral operators are also considered.

2. Inclusion properties involving the operator $I_{\lambda, \mu}^{n}$. The following lemmas will be required in our investigation.

Lemma 1 ([4]). Let $\phi$ be convex univalent in $U$ with $\phi(0)=1$ and $\operatorname{Re}\{\mu \phi(z)+\nu\}>0(\mu, \nu \in C)$. If $p$ is analytic in $U$ with $p(0)=1$, then

$$
p(z)+\frac{z p^{\prime}(z)}{\mu p(z)+\nu} \prec \phi(z) \quad(z \in U)
$$

implies that

$$
p(z) \prec \phi(z) \quad(z \in U) .
$$

Lemma 2 ([8]). Let $\phi$ be convex univalent in $U$ and $w$ be analytic in $U$ with $\operatorname{Re}\{w(z)\} \geq 0$. If $p$ is analytic in $U$ and $p(0)=\phi(0)$, then

$$
p(z)+w(z) z p^{\prime}(z) \prec \phi(z) \quad(z \in U)
$$

implies that

$$
p(z) \prec \phi(z) \quad(z \in U) .
$$

At first, with the help of Lemma 1, we obtain the following theorem.

Theorem 1. Let $n \in N_{0}, \lambda>0, \mu \geq 1$ and $\operatorname{Re}\left\{(1-\eta) \phi(z)+\frac{1}{\lambda}-1+\eta\right\}>0$. Then we have

$$
S_{\lambda, \mu+1}^{n}(\eta ; \phi) \subset S_{\lambda, \mu}^{n}(\eta ; \phi) \subset S_{\lambda, \mu}^{n+1}(\eta ; \phi)
$$

$(0 \leq \eta<1 ; \phi \in S)$.

Proof. First of all, we will show that

$$
S_{\lambda, \mu+1}^{n}(\eta ; \phi) \subset S_{\lambda, \mu}^{n}(\eta ; \phi) .
$$

Let $f \in S_{\lambda, \mu+1}^{n}(\eta ; \phi)$ and put

$$
p(z)=\frac{1}{1-\eta}\left(\frac{z\left(I_{\lambda, \mu}^{n} f(z)\right)^{\prime}}{I_{\lambda, \mu}^{n} f(z)}-\eta\right),
$$


where $p(z)$ is analytic in $U$ with $p(0)=1$. Using the identity (1.6) in (2.1), we obtain

$$
\mu \frac{I_{\lambda, \mu+1}^{n} f(z)}{I_{\lambda, \mu}^{n} f(z)}=(1-\eta) p(z)+\mu-1+\eta .
$$

Differentiating (2.2) logarithmically with respect to $z$ and multiplying by $z$, we obtain

$$
\frac{1}{1-\eta}\left(\frac{z\left(I_{\lambda, \mu+1}^{n} f(z)\right)^{\prime}}{I_{\lambda, \mu+1}^{n} f(z)}-\eta\right)=p(z)+\frac{z p^{\prime}(z)}{(1-\eta) p(z)+\mu-1+\eta}
$$

$(z \in U)$. Applying Lemma 1 to $(2.3)$, we see that $p(z) \prec \phi(z)$, that is, $f \in S_{\lambda, \mu}^{n}(\eta ; \phi)$.

To prove the second part, let $f \in S_{\lambda, \mu}^{n}(\eta ; \phi)$ and put

$$
h(z)=\frac{1}{1-\eta}\left(\frac{z\left(I_{\lambda, \mu}^{n+1} f(z)\right)^{\prime}}{I_{\lambda, \mu}^{n+1} f(z)}-\eta\right),
$$

where $h$ is analytic in $U$ with $h(0)=1$. Then, by using the arguments similar to these detailed above with (1.5), it follows that $h \prec \phi(z \in U)$, which implies that $f \in S_{\lambda, \mu}^{n+1}(\eta ; \phi)$. This completes the proof of Theorem 1.

Theorem 2. Let $n \in N_{0}, \lambda>0$ and $\mu \geq 1$. Then we have

$$
K_{\lambda, \mu+1}^{n}(\eta ; \phi) \subset K_{\lambda, \mu}^{n}(\eta ; \phi) \subset K_{\lambda, \mu}^{n+1}(\eta ; \phi)
$$

$(0 \leq \eta<1 ; \phi \in S)$.

Proof. Applying (1.7) and Theorem 1, we observe that

$$
\begin{aligned}
f(z) \in K_{\lambda, \mu+1}^{n}(\eta ; \phi) & \Longleftrightarrow I_{\lambda, \mu+1}^{n} f(z) \in K(\eta ; \phi) \\
& \Longleftrightarrow z\left(I_{\lambda, \mu+1}^{n} f(z)\right)^{\prime} \in S^{*}(\eta ; \phi) \\
& \Longleftrightarrow I_{\lambda, \mu+1}^{n}\left(z f^{\prime}(z)\right) \in S^{*}(\eta ; \phi) \\
& \Longleftrightarrow z f^{\prime}(z) \in S_{\lambda, \mu+1}^{n}(\eta ; \phi) \\
& \Longleftrightarrow z f^{\prime}(z) \in S_{\lambda, \mu}^{n}(\eta ; \phi) \\
& \Longleftrightarrow I_{\lambda, \mu}^{n}\left(z f^{\prime}(z)\right)^{*} \in S^{*}(\eta ; \phi) \\
& \Longleftrightarrow z\left(I_{\lambda, \mu}^{n} f(z)\right)^{\prime} \in S^{*}(\eta ; \phi) \\
& \Longleftrightarrow I_{\lambda, \mu}^{n} f(z) \in K(\eta ; \phi) \\
& \Longleftrightarrow f(z) \in K_{\lambda, \mu}^{n}(\eta ; \phi)
\end{aligned}
$$

and

$$
\begin{aligned}
f(z) \in K_{\lambda, \mu}^{n}(\eta ; \phi) & \Longleftrightarrow z f^{\prime}(z) \in S_{\lambda, \mu}^{n}(\eta ; \phi) \\
& \Longleftrightarrow z f^{\prime}(z) \in S_{\lambda, \mu}^{n+1}(\eta ; \phi) \\
& \Longleftrightarrow z\left(I_{\lambda, \mu}^{n+1} f(z)\right)^{\prime} \in S^{*}(\eta ; \phi) \\
& \Longleftrightarrow I_{\lambda, \mu}^{n+1} f(z) \in K(\eta ; \phi) \\
& \Longleftrightarrow f(z) \in K_{\lambda, \mu}^{n+1}(\eta ; \phi),
\end{aligned}
$$


which evidently proves the theorem.

Remark. Taking

$$
\phi(z)=\left(\frac{1+A z}{1+B z}\right)^{\alpha} \quad(-1 \leq B<A \leq 1 ; 0<\alpha \leq 1 ; z \in U)
$$

in Theorems 1 and 2, we have the following corollary.

Corollary 1. Let $n \in N_{0}, \lambda>0$ and $\mu \geq 1$. Then we have

$$
\begin{aligned}
& S_{\lambda, \mu+1}^{n}(\eta ; A, B ; \alpha) \subset S_{\lambda, \mu}^{n}(\eta ; A, B ; \alpha) \subset S_{\lambda, \mu}^{n+1}(\eta ; A, B ; \alpha) \\
& (0 \leq \eta<1 ;-1 \leq B<A \leq 1 ; 0<\alpha \leq 1) \text {, and } \\
& \quad K_{\lambda, \mu+1}^{n}(\eta ; A, B ; \alpha) \subset K_{\lambda, \mu}^{n}(\eta ; A, B ; \alpha) \subset K_{\lambda, \mu}^{n+1}(\eta ; A, B ; \alpha) \\
& (0 \leq \eta<1 ;-1 \leq B<A \leq 1 ; 0<\alpha \leq 1) .
\end{aligned}
$$

Next, by using Lemma 2, we obtain the following inclusion relation for the class $C_{\lambda, \mu}^{n}(\eta, \delta ; \phi, \psi)$.

Theorem 3. Let $n \in N_{0}, \lambda>0$ and $\mu \geq 1$. Then we have

$$
C_{\lambda, \mu+1}^{n}(\eta, \delta ; \phi, \psi) \subset C_{\lambda, \mu}^{n}(\eta, \delta ; \phi, \psi) \subset C_{\lambda, \mu}^{n+1}(\eta, \delta ; \phi, \psi)
$$

$(0 \leq \eta, \delta<1 ; \phi, \psi \in S)$.

Proof. We begin by proving that

$$
C_{\lambda, \mu+1}^{n}(\eta, \delta ; \phi, \psi) \subset C_{\lambda, \mu}^{n}(\eta, \delta ; \phi, \psi) .
$$

Let $f \in C_{\lambda, \mu+1}^{n}(\eta, \delta ; \phi, \psi)$. Then, in view of the definition of the class $C_{\lambda, \mu+1}^{n}(\eta, \delta ; \phi, \psi)$, there exists a function $g \in S_{\lambda, \mu+1}^{n}(\eta ; \phi)$ such that

$$
\frac{1}{1-\delta}\left(\frac{z\left(I_{\lambda, \mu+1}^{n} f(z)\right)^{\prime}}{I_{\lambda, \mu+1}^{n} g(z)}-\delta\right) \prec \psi(z) \quad(z \in U) .
$$

Now let

$$
p(z)=\frac{1}{1-\delta}\left(\frac{z\left(I_{\lambda, \mu}^{n} f(z)\right)^{\prime}}{I_{\lambda, \mu}^{n} g(z)}-\delta\right),
$$

where $p(z)$ is analytic in $U$ with $p(0)=1$. Using (1.6), we have

$$
[(1-\delta) p(z)+\delta] I_{\lambda, \mu}^{n} g(z)+(\mu-1) I_{\lambda, \mu}^{n} f(z)=\mu I_{\lambda, \mu+1}^{n} f(z) .
$$

Differentiating (2.4) with respect to $z$ and multiplying by $z$, we obtain

$$
\begin{aligned}
(1-\delta) z p^{\prime}(z) I_{\lambda, \mu}^{n} g(z) & +[(1-\delta) p(z)+\delta] z\left(I_{\lambda, \mu}^{n} g(z)\right)^{\prime} \\
& =\mu z\left(I_{\lambda, \mu+1}^{n} f(z)\right)^{\prime}-(\mu-1) z\left(I_{\lambda, \mu}^{n} f(z)\right)^{\prime} .
\end{aligned}
$$


Since $g(z) \in S_{\lambda, \mu+1}^{n}(\eta ; \phi)$, by Theorem $1, g \in S_{\lambda, \mu}^{n}(\eta ; \phi)$. Let

$$
q(z)=\frac{1}{1-\eta}\left(\frac{z\left(I_{\lambda, \mu}^{n} g(z)\right)^{\prime}}{I_{\lambda, \mu}^{n} g(z)}-\eta\right) .
$$

Then, using (1.6) once again, we have

$$
\mu \frac{I_{\lambda, \mu+1}^{n} g(z)}{I_{\lambda, \mu}^{n} g(z)}=(1-\eta) q(z)+\mu-1+\eta .
$$

From (2.5) and (2.6), we obtain

$$
\frac{1}{1-\delta}\left(\frac{z\left(I_{\lambda, \mu+1}^{n} f(z)\right)^{\prime}}{I_{\lambda, \mu+1}^{n} g(z)}-\delta\right)=p(z)+\frac{z p^{\prime}(z)}{(1-\eta) q(z)+\mu-1+\eta} .
$$

Since $0 \leq \eta<1, \mu \geq 1$ and $q(z) \prec \phi(z)(z \in U)$, we have

$$
\operatorname{Re}\{(1-\eta) q(z)+\mu-1+\eta\}>0 \quad(z \in U) .
$$

Hence, applying Lemma 2, we can show that $p(z) \prec \psi(z)$, so that $f \in$ $C_{\lambda, \mu}^{n}(\eta, \delta ; \phi, \psi)$.

For the second part, by using the arguments similar to these detailed above with (1.5), we obtain

$$
C_{\lambda, \mu}^{n}(\eta, \delta ; \phi, \psi) \subset C_{\lambda, \mu}^{n+1}(\eta, \delta ; \phi, \psi) .
$$

This completes the proof of Theorem 3 .

3. Inclusion properties involving the integral operator $\boldsymbol{F}_{\boldsymbol{c}}$. In this section, we consider the generalized Libera integral operator $F_{c}$ (see [2], [6] and [9]) defined by

$$
F_{c}(f)=F_{c}(f)(z)=\frac{c+1}{z^{c}} \int_{0}^{z} t^{c-1} f(t) d t \quad(f \in A ; c>-1) .
$$

We first prove the following theorem.

Theorem 4. Let $c \geq 0, n \in N_{0}, \lambda>0$ and $\mu>0$. If $f \in S_{\lambda, \mu}^{n}(\eta ; \phi)$ $(0 \leq \eta<1 ; \phi \in S)$, then we have $F_{c}(f) \in S_{\lambda, \mu}^{n}(\eta ; \phi)(0 \leq \eta<1 ; \phi \in S)$.

Proof. Let $f \in S_{\lambda, \mu}^{n}(\eta ; \phi)$ and put

$$
p(z)=\frac{1}{1-\eta}\left(\frac{z\left(I_{\lambda, \mu}^{n} F_{c}(f)(z)\right)^{\prime}}{I_{\lambda, \mu}^{n} F_{c}(f)(z)}-\eta\right)
$$


where $p(z)$ is analytic in $U$ with $p(0)=1$. From (3.1), we have

$$
z\left(I_{\lambda, \mu}^{n} F_{c}(f)(z)\right)^{\prime}=(c+1) I_{\lambda, \mu}^{n} f(z)-c I_{\lambda, \mu}^{n} F_{c}(f)(z) .
$$

Then, by using (3.2) and (3.3), we have

$$
(c+1) \frac{I_{\lambda, \mu}^{n} f(z)}{I_{\lambda, \mu}^{n} F_{c}(f)(z)}=(1-\eta) p(z)+c+\eta .
$$

Differentiating (3.4) logarithmically with respect to $z$ and multiplying by $z$, we obtain

$$
p(z)+\frac{z p^{\prime}(z)}{(1-\eta) p(z)+c+\eta}=\frac{1}{1-\eta}\left(\frac{z\left(I_{\lambda, \mu}^{n} f(z)\right)^{\prime}}{I_{\lambda, \mu}^{n} f(z)}-\eta\right) \quad(z \in U) .
$$

Hence from Lemma 1, we conclude that $p(z) \prec \phi(z)(z \in U)$, which implies $F_{c}(f) \in S_{\lambda, \mu}^{n}(\eta ; \phi)$.

Next, we derive an inclusion property involving $F_{c}$, which is given by the following theorem.

Theorem 5. Let $c \geq 0, \lambda>0, n \in N_{0}$ and $\mu>0$. If $f \in K_{\lambda, \mu}^{n}(\eta ; \phi)$ $(0 \leq \eta<1 ; \phi \in S)$, then we have

$$
F_{c}(f) \in K_{\lambda, \mu}^{n}(\eta ; \phi) \quad(0 \leq \eta<1 ; \phi \in S) .
$$

Proof. By applying Theorem 4, we have

$$
\begin{aligned}
f(z) \in K_{\lambda, \mu}^{n}(\eta ; \phi) & \Longleftrightarrow z f^{\prime}(z) \in S_{\lambda, \mu}^{n}(\eta ; \phi) \\
& \Longleftrightarrow F_{c}\left(z f^{\prime}(z)\right) \in S_{\lambda, \mu}^{n}(\eta ; \phi) \\
& \Longleftrightarrow z\left(F_{c}(f)(z)\right)^{\prime} \in S_{\lambda, \mu}^{n}(\eta ; \phi) \\
& \Longleftrightarrow F_{c}(f)(z) \in K_{\lambda, \mu}^{n}(\eta ; \phi)
\end{aligned}
$$

which proves Theorem 5 .

From Theorems 4 and 5, we have the following corollary.

Corollary 2. Let $c \geq 0, \lambda>0, n \in N_{0}$ and $\mu>0$. If $f(z)$ belongs to the class $S_{\lambda, \mu}^{n}(\eta ; A, B ; \alpha)$ (or $\left.K_{\lambda, \mu}^{n}(\eta ; A, B ; \alpha)\right)(0 \leq \eta<1 ;-1 \leq B<$ $A \leq 1 ; 0<\alpha \leq 1)$, then $F_{c}(f)$ belongs to the class $S_{\lambda, \mu}^{n}(\eta ; A, B ; \alpha)$ (or $\left.K_{\lambda, \mu}^{n}(\eta ; A, B ; \alpha)\right)(0 \leq \eta<1 ;-1 \leq B<A \leq 1 ; 0<\alpha \leq 1)$.

Finally, we prove the following theorem.

Theorem 6. Let $c \geq 0, \lambda>0, n \in N_{0}$ and $\mu>0$. If $f \in C_{\lambda, \mu}^{n}(\eta, \delta ; \phi, \psi)$ $(0 \leq \eta, \delta<1 ; \phi, \psi \in S)$, then we have $F_{c}(f) \in C_{\lambda, \mu}^{n}(\eta, \delta ; \phi, \psi)(0 \leq \eta$, $\delta<1 ; \phi, \psi \in S)$. 
Proof. Let $f \in C_{\lambda, \mu}^{n}(\eta, \delta ; \phi, \psi)$. Then, in view of the definition of the class $C_{\lambda, \mu}^{n}(\eta, \delta ; \phi, \psi)$, there exists a function $g \in S_{\lambda, \mu}^{n}(\eta ; \phi)$ such that

$$
\frac{1}{1-\delta}\left(\frac{z\left(I_{\lambda, \mu}^{n} f(z)\right)^{\prime}}{I_{\lambda, \mu}^{n} g(z)}-\delta\right) \prec \psi(z) \quad(z \in U) .
$$

Thus, we put

$$
p(z)=\frac{1}{1-\delta}\left(\frac{z\left(I_{\lambda, \mu}^{n} F_{c}(f)(z)\right)^{\prime}}{I_{\lambda, \mu}^{n} F_{c}(g)(z)}-\delta\right),
$$

where $p(z)$ is analytic in $U$ with $p(0)=1$. Since $g(z) \in S_{\lambda, \mu}^{n}(\eta ; \phi)$, we see from Theorem 4 that $F_{c}(g) \in S_{\lambda, \mu}^{n}(\eta ; \phi)$. Using (3.3), we have

$$
[(1-\delta) p(z)+\delta] I_{\lambda, \mu}^{n} F_{c}(g)(z)+c I_{\lambda, \mu}^{n} F_{c}(f)(z)=(c+1) I_{\lambda, \mu}^{n} f(z) .
$$

Differentiating (3.6) with respect to $z$ and multiplying by $z$, we obtain $(c+1) \frac{z\left(I_{\lambda, \mu}^{n} f(z)\right)^{\prime}}{I_{\lambda, \mu}^{n} F_{c}(g)(z)}=[(1-\delta) p(z)+\delta][(1-\eta) q(z)+c+\eta]+(1-\delta) z p^{\prime}(z)$, where

$$
q(z)=\frac{1}{1-\eta}\left(\frac{z\left(I_{\lambda, \mu}^{n} F_{c}(g)(z)\right)^{\prime}}{I_{\lambda, \mu}^{n} F_{c}(g)(z)}-\eta\right)
$$

Hence, we have

$$
\frac{1}{1-\delta}\left(\frac{z\left(I_{\lambda, \mu}^{n} f(z)\right)^{\prime}}{I_{\lambda, \mu}^{n} g(z)}-\delta\right)=p(z)+\frac{z p^{\prime}(z)}{(1-\eta) q(z)+c+\eta} .
$$

The remaining part of the proof in Theorem 6 is similar to that of Theorem 3 and so we omit it.

Acknowledgements. The authors would like to thank the referees of the paper for their helpful suggestions.

\section{REFERENCES}

[1] Al-Oboudi, F. M., On univalent functions defined by a generalized Sălăgean operator, Internat. J. Math. Math. Sci. 27 (2004), 1429-1436.

[2] Bernardi, S. D., Convex and starlike univalent functions, Trans. Amer. Math. Soc. 35 (1969), 429-446.

[3] Choi, J. H., Saigo, M. and Srivastava, H. M., Some inclusion properties of a certain family of integral operators, J. Math. Anal. Appl. 276 (2002), 432-445.

[4] Eenigenburg, P., Miller, S. S., Mocanu, P. T. and Reade, M. O., On a Briot-Bouquet differential subordination, General inequalities, 3 (Oberwolfach, 1981), 339-348, Internat. Schriftenreihe Numer. Math., 64, Birkhäuser, Basel, 1983. 
[5] Kim, Y. C., Choi, J. H. and Sugawa, T., Coefficient bounds and convolution properties for certain classes of close-to-convex functions, Proc. Japan Acad. Ser. A Math. Sci. 76 (2000), 95-98.

[6] Libera, R. J., Some classes of regular univalent functions, Proc. Amer. Math. Soc. 16 (1965), 755-758.

[7] Ma, W. C., Minda, D., An internal geometric characterization of strongly starlike functions, Ann. Univ. Mariae Curie-Skłodowska Sect. A 45 (1991), 89-97.

[8] Miller, S. S., Mocanu, P. T., Differential subordinations and univalent functions, Michigan Math. J. 28 (1981), 157-171.

[9] Owa, S., Srivastava, H. M., Some applications of the generalized Libera operator, Proc. Japan Acad. Ser. A Math. Sci. 62 (1986), 125-128.

[10] Sălăgean, G. S., Subclasses of univalent functions, Complex analysis - fifth Romanian-Finnish seminar, Part 1 (Bucharest, 1981), 362-372, Lecture Notes in Math., 1013, Springer, Berlin, 1983.

[11] Srivastava, H. M., Owa, S. (Editors), Current Topics in Analytic Function Theory, World Scientific Publishing Company, Singapore, 1992.

M. K. Aouf

Department of Mathematics

Faculty of Science

Mansoura University

Mansoura 35516

Egypt

e-mail: mkaouf 127@yahoo.com

A. O. Mostafa

Department of Mathematics

Faculty of Science

Mansoura University

Mansoura 35516

Egypt

e-mail: adelaeg254@yahoo.com
A. Shamandy

Department of Mathematics

Faculty of Science

Mansoura University

Mansoura 35516

Egypt

e-mail: shamandy16@hotmail.com

S. M. Madian

Department of Mathematics

Faculty of Science

Mansoura University

Mansoura 35516

Egypt

e-mail: samar_math@yahoo.com

Received April 11, 2009 